\title{
Comparative venom gland transcriptome analysis of the scorpion Lychas mucronatus reveals intraspecific toxic gene diversity and new venomous components
}

\author{
Zhao Ruiming ${ }^{\dagger}$, Ma Yibao ${ }^{\dagger}$, He Yawen, Di Zhiyong, Wu Yingliang, Cao Zhijian*, Li Wenxin ${ }^{*}$
}

\begin{abstract}
Background: Lychas mucronatus is one scorpion species widely distributed in Southeast Asia and southern China. Anything is hardly known about its venom components, despite the fact that it can often cause human accidents. In this work, we performed a venomous gland transcriptome analysis by constructing and screening the venom gland cDNA library of the scorpion Lychas mucronatus from Yunnan province and compared it with the previous results of Hainan-sourced Lychas mucronatus.

Results: A total of sixteen known types of venom peptides and proteins are obtained from the venom gland cDNA library of Yunnan-sourced Lychas mucronatus, which greatly increase the number of currently reported scorpion venom peptides. Interestingly, we also identified nineteen atypical types of venom molecules seldom reported in scorpion species. Surprisingly, the comparative transcriptome analysis of Yunnan-sourced Lychas mucronatus and Hainan-sourced Lychas mucronatus indicated that enormous diversity and vastly abundant difference could be found in venom peptides and proteins between populations of the scorpion Lychas mucronatus from different geographical regions.
\end{abstract}

Conclusions: This work characterizes a large number of venom molecules never identified in scorpion species. This result provides a comparative analysis of venom transcriptomes of the scorpion Lychas mucronatus from different geographical regions, which thoroughly reveals the fact that the venom peptides and proteins of the same scorpion species from different geographical regions are highly diversified and scorpion evolves to adapt a new environment by altering the primary structure and abundance of venom peptides and proteins.

\section{Background}

More than 400 million years of evolution does not make the scorpions alter their morphology. However, although this one of the oldest arachnid is highly conserved in shape all along, they occupy vast territory of the world from Africa to Asia, Australia and America. Such powerful adaptability mostly owes to their highly specialized venom apparatus which consists of the vesicle holding a pair of venom glands connected to the stinger used to inject the venom [1]. The extant scorpions can be phylogenetically divided into 14 families based on a

\footnotetext{
* Correspondence: zjcao@whu.edu.cn; liwxlab@whu.edu.cn † Contributed equally

State Key Laboratory of Virology, College of Life Sciences, Wuhan University, Wuhan, 430072, People's Republic of China
}

morphological cladistic analysis, among which the Buthidae is considered to be the largest and the most medically important family [2]. As a result, this enormous family has attracted the biggest scientific interest and been extensively studied. Till now, approximate 800 scorpion species have been classified into the Buthidae family. Previous study confirmed that each one of the species may typically contain more than one hundred different peptides in venom ranging in mass from 1,000-9,000 $\mathrm{Da}$ [3-5]. Among the venom peptides in this range, two classes are most regarded. One of them is small neurotoxic proteins that recognize ion channels and receptors in membranes of excitable cells, and thus toxic to different organisms $[6,7]$. The other is antimicrobial peptides 
which are important defensive molecules of the ancient innate immunity [8].

It is acknowledged that the Buthidae family possesses a very different venom arsenal comparing to other nonButhidae families [9]. Moreover, even within the Buthidae family, vast abundance difference can be observed in venom compositions between genus to genus, different species within a genus and individuals within a species [10-12]. As a result, previous study estimated that approximately 150,000 distinct polypeptides presented in about 1500 known scorpion species in the world [13]. Numerous peptides in scorpion venoms are a big treasury waiting for exploitation. Proteomic and transcriptomic approaches have already helped us to draw a rough picture of the molecular diversity of the scorpion venom components [14]. To the best of our knowledge, the transcriptomic approach is more effective in getting an overview of scorpion venom. Not only because it can reflect the biological processes inside the venom gland cells, but also provides clues to the research of evolutionary path leading to scorpion toxin diversification directly [4].

The family Buthidae has drawn most of attention because of its medical importance. Beyond all doubt, the venom peptides from family Buthidae will play an important role in studying biological systems and be a treasure for use in drug development. However, up to date, research interests mostly concentrate on a few genera in this family such as Androctonus [15], Buthus [16], Mesobuthus [17], Parabuthus [18], Centruroides [19], or Tityus [20]. In a sort of sense, other almost eighty genera are totally ignored, despite whose venom components are extremely attractive. Lychas mucronatus belonging to the genus Lychas of the family Buthidae is one of these scorpion species lacking of concern. Lychas mucronatus is widely distributed in Southeast Asia and southern China including Hainan island [21]. This small-sized venomous animal can be found easily in the suburbs of Yunnan, Guangxi and Hainan provinces [22]. Recently, our group collected one population of Lychas mucronatus from Hainan province. We performed its venom transcriptome analysis, and some new toxins were identified [23-25]. After that, we also obtained another population of the scorpion Lychas mucronatus from Yunnan province (Figure 1) which subsequently identified to be the same species with Hainan-sourced population according to morphological identification. It provided an opportunity to give a faithful and particular investigation on intraspecific diversity of scorpion venom

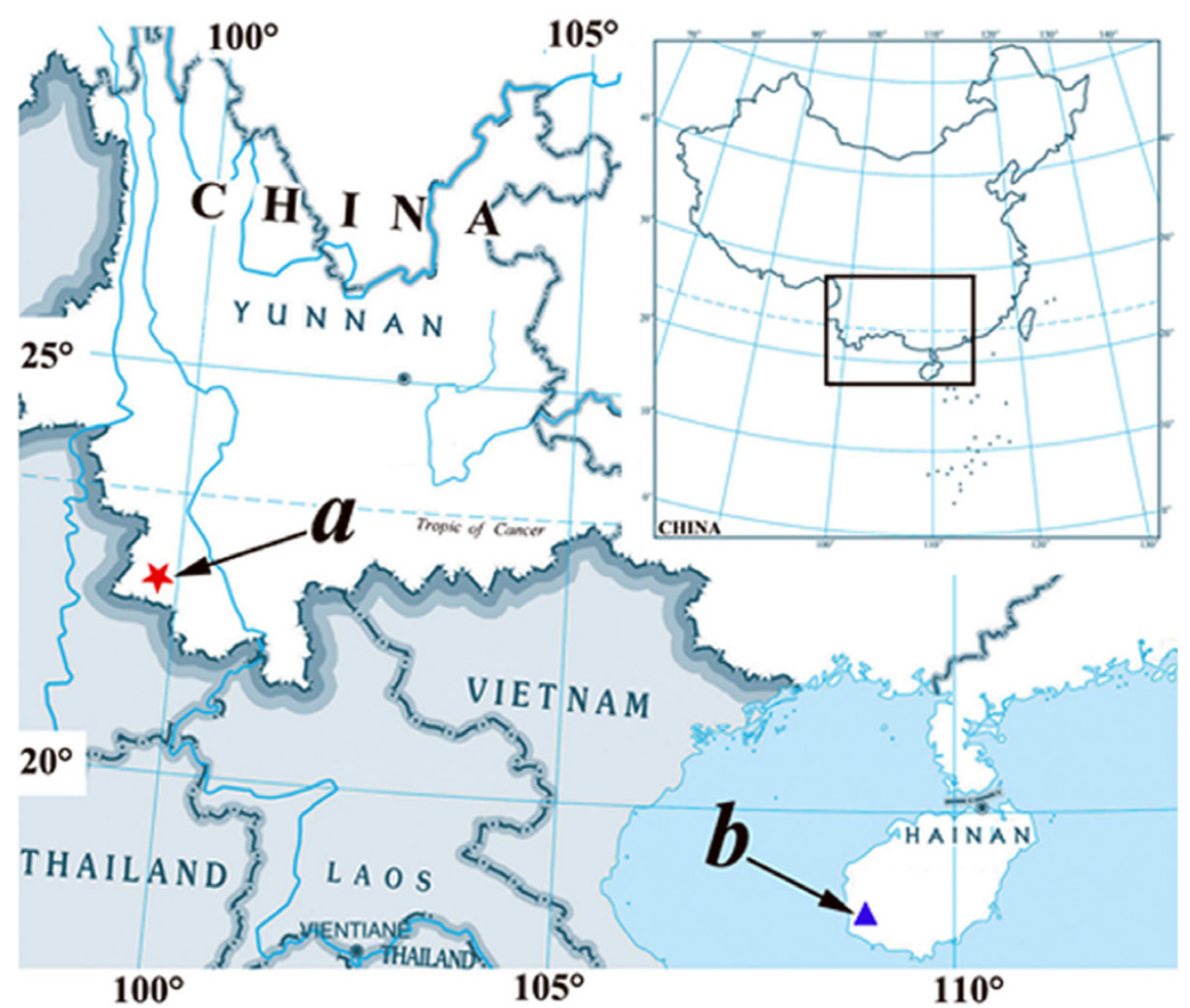

Figure 1 Geographical locations of two populations of Lychas mucronatus. The marker (a) shows Shidian county in Yunnan province and (b) shows Janfeng mountain in Hainan island. 
peptides exist at the transcript and gene levels. Although previous researches have demonstrated that there are intraspecific molecular diversity exiting in geographic isolated scorpion populations, these researches used mtDNA phylogenetic reconstruction or limited proteomic information to elucidate how the subspecies of scorpion evolved accompanied with biogeographic events [26,27]. Comprehensive and accurate information about intraspecific variation of scorpion venom peptides and proteins is still unrevealed. In this work, we constructed a non-normalized cDNA Library and carried an EST approach to overview the transcriptome of the venom gland of the scorpion Lychas mucronatus from Yunnan province. A great deal of venom peptides and proteins belonging to known or atypical toxin types were identified. Most high abundance toxin types were compared with previous results conducted on Hainan-sourced population. This work provides a comprehensive comparative analysis of venom transcriptomes of two Lychas mucronatus populations. The results reveal the fact that both the primary structure and abundance of venom peptides are quite different in the same scorpion species from different geographical regions. Besides, transcriptomic comparison between two populations of Lychas mucronatus also provides some clues to understand how scorpion is reorganizing its venom toxins to adapt a new environment in evolutionary development.

\section{Results}

\section{Morphological comparison of two Lychas mucronatus} populations

Lychas mucronatus is one scorpion species widely distributed in Southeast Asia. In China, they chiefly exist in Yunnan, Guangxi and Hainan provinces. Our group gets two populations of the scorpion Lychas mucronatus successively from Hainan and Yunnan provinces with distinct ecotopes (Figure 1). Based on Kovařík and Zhu's description [21,22], the adults of Lychas mucronatus can be easily recognized based on coloration and morphological characteristics as following: (1) Generally, Lychas mucronatus is $40-65 \mathrm{~mm}$ long, carapace, mesosomal tergites and legs are yellow and blotched, metasoma segments are yellow to yellowish-brown from base to terminal, pedipalp, patella is predominantly dark, pedipalps manus is bright yellow with sparse, minute black spots and fingers are dark yellow brown; (2) Pedipalp fingers of males are curved, whereas those of female are straight; (3) Second segment of metasoma has 10 keels, and the third metasomal segment has 8 keels; (4) Sixth cutting edge on movable fingers of pedipalps has 3 granules; (5) Pectinal teeth number is 16-26 (frequently 19-23); (6) Metasoma of males has the same length as that of females.
According to microscopic observation, both scorpion populations we collected possess the same characters mentioned above. So we convinced that two scorpion populations obtained from two provinces actually belong to the same species Lychas mucronatus.

\section{EST sequencing and clustering}

The Yunnan-sourced Lychas mucronatus cDNA library was constructed with the same methods as Hainansourced Lychas mucronatus [23]. Both of libraries are not amplified, their clone number rates of different types of venom peptides and proteins should reflect the actual transcriptive abundance in original biological samples. The Yunnan-sourced Lychas mucronatus cDNA library possessed a $2.6 \times 10^{6} \mathrm{cfu} / \mathrm{ml}$ titer with more than $99 \%$ recombinant efficiency. In order to get more types of peptides and proteins, we selected 1000 random colonies for sequencing which have a total of 738 readable sequences. These EST sequences were then submitted into the dbEST (accession numbers: GT028570-GT029307). After processed by EGassembler online bioinformatics service [28], 738 ESTs are assembled as 309 singletons and 71 contigs consisting of two or more ESTs (Figure 2).

We compared the consensual cluster sequences against SWISS-PROT and GenBank NCBI databases by BLAST algorithms for a functional classification of these unique sequences. 189 clusters (488 ESTs) showed homology to existent peptides or proteins (Expect value <e-4), whereas the other 191 clusters (250 ESTs) hadn't good matches (Table 1). Among these "match" sequences, 83 clusters (414 ESTs) are deduced to be secretory peptides and proteins. These putative toxins and secretory venom components represent nearly $56 \%$ of total ESTs. It is worth noticing that neurotoxins account for $20 \%$ of the whole transcripts and peptides putative to have the antimicrobial function account for $12 \%$. For those "non-match" ESTs, the longest ORFs were generally predicted and screened for possible signal peptides. The result indicated that 25 clusters (79 ESTs) are supposed to possess a signal peptide. This part of non-matched clones obviously a possible source for new venom peptides. 127 clusters (128 ESTs) don't have signal peptide and 39 clusters (43 ESTs) haven't ORF found. The total unassigned ESTs account for 33\% which is similar to the results of other transcriptome studies [4,29]. Particular information about bioinformatical analysis of Hainan-sourced Lychas mucronatus cDNA library can be got from our submitted work "Evolution of the scorpion venom arsenal inferred by comparative transcriptome and molecular phylogeny analyses" (unpublished data). The distribution of all ESTs in both populations was depicted in Figure 3. 


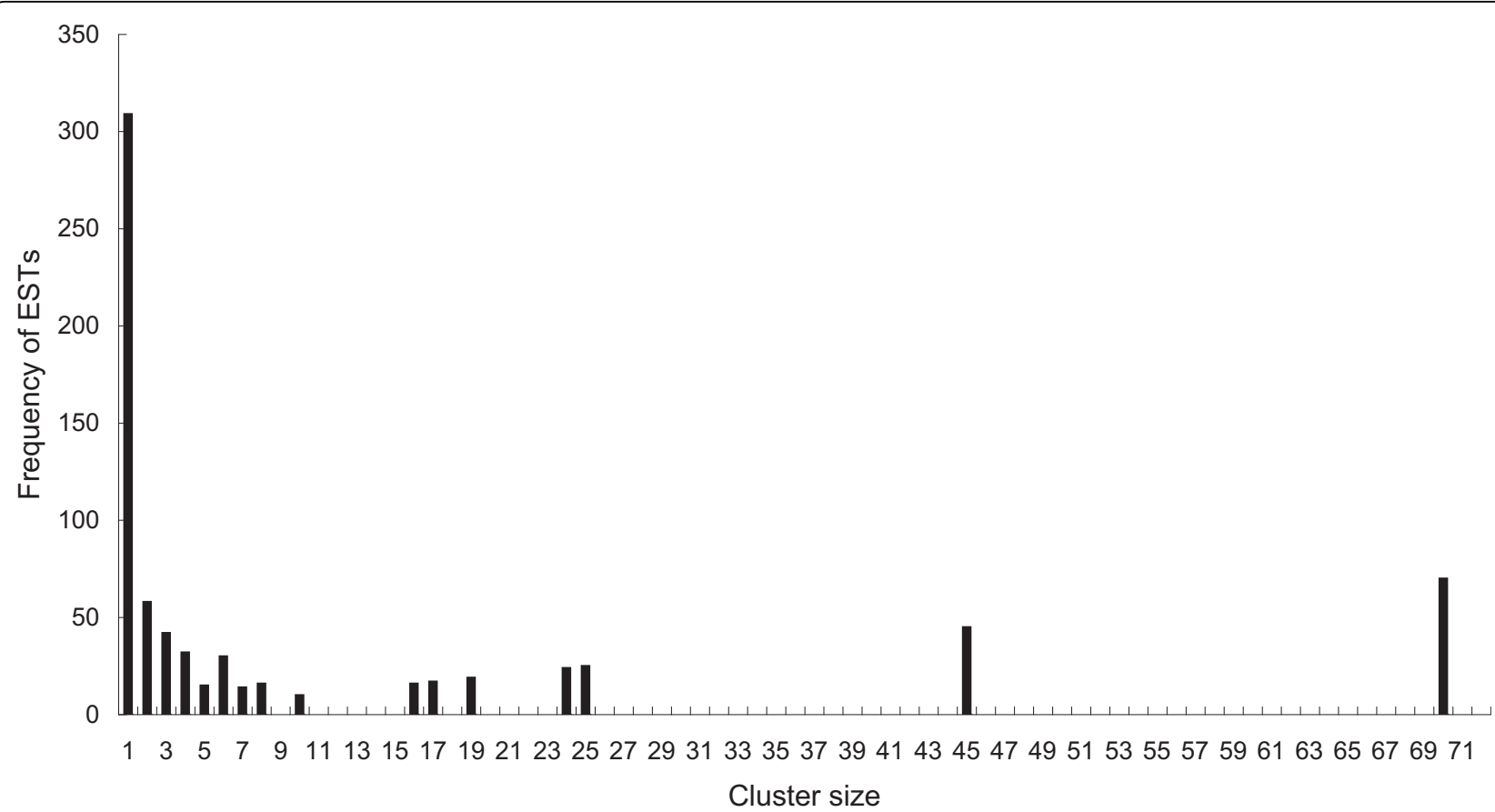

Figure 2 ESTs distribution by cluster size. For instance, there are 8 clusters of size 4, accounting for a sum of 32 ESTs.

\section{Known toxin types}

The "-ome" approaches have already helped to reveal a great number of scorpion venom toxin types $[4,10,30]$. Most of these molecules are named as known toxin types because they have been identified for function or at least their primary structures were reported in literature $[3,31,32]$. However, there always have new scorpion venom toxins with special primary structures and unrevealed functions been reported continuously [33]. This part of toxins is named as atypical toxin types. From the venom gland cDNA library of the scorpion Lychas mucronatus collected from Yunnan province, sixteen known toxin types were obtained. They are encoded by 342 ESTs (57 clusters), accounting for approximately $46 \%$ of the total venom gland transcripts. All these sequences possess signal peptides. Most of these types have been identified at the protein level, whereas a few of them are only found at the transcript level. The particular comparison of these known toxin types from Yunnan Lychas mucronatus and previously identified known toxin types from Hainan Lychas mucronatus will be detailed in subjects below (Table 2).

NaTx (toxins specific for sodium channels)

Till now, NaTx were only found in the venom of the family Buthidae except one peptide phaiodotoxin from the family Iuridae [34]. They are 6500-8500 Da (58-76 residues) polypeptides showing the conserved structure core constrained by three disulfide bridges: two disulfides link the $\alpha$-helix to the anti-parallel $\beta$-sheet and the third disulfide links the $\beta$-sheet to an extended segment preceding the helix. But the fourth disulfide bridge, also called wrapper disulfide bridge [35], varies in position, and sometimes is even lost. These structure-conserved 'long-chain' peptides are considered to be the primary causes of the neurotoxic symptoms induced by scorpion envenomation.

Table 1 Distribution of $\mathbf{3 8 0}$ clusters assembled from the scorpion Lychas mucronatus collected from Yunnan province

\begin{tabular}{|c|c|c|c|}
\hline Category & $\begin{array}{c}\text { Secretory } \\
\text { (clusters/ESTs) }\end{array}$ & $\begin{array}{l}\text { Non-secretory } \\
\text { (clusters/ESTs) }\end{array}$ & $\begin{array}{c}\text { Non ORF } \\
\text { (clusters/ESTs) }\end{array}$ \\
\hline \multicolumn{4}{|l|}{ Matching sequences } \\
\hline Similar to venom peptide transcripts & $50(325)$ & & \\
\hline Not similar to venom peptide transcripts & $8(10)$ & 131(153) & \\
\hline Non-matching sequences & $25(79)$ & $127(128)$ & $39(43)$ \\
\hline Total & $83(414)$ & 258(281) & $39(43)$ \\
\hline
\end{tabular}




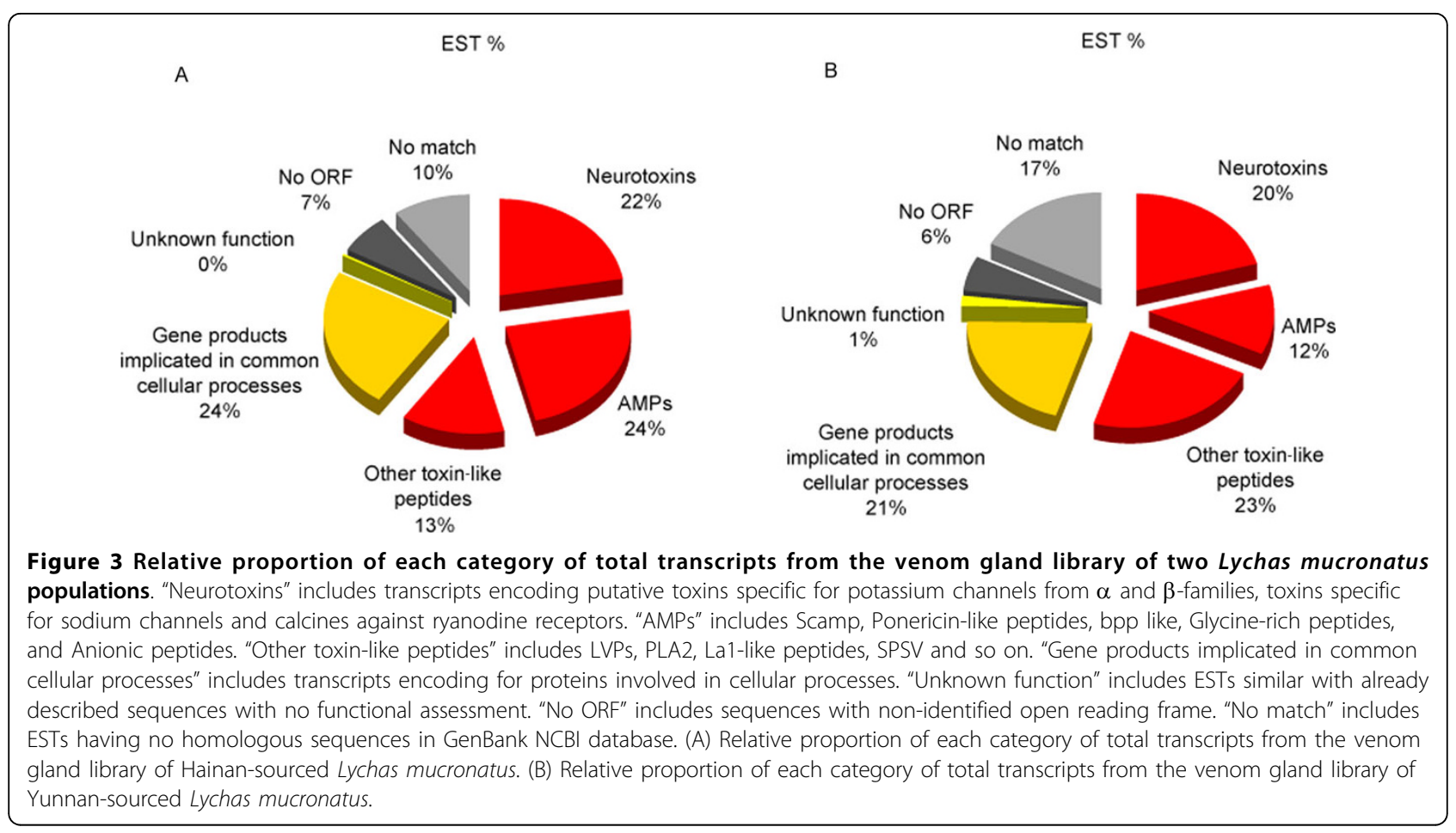

Table 2 Comparison of abundance of different toxin types between two populations of Lychas mucronatus

\begin{tabular}{|c|c|c|c|c|}
\hline \multirow[t]{3}{*}{ Toxin types } & \multicolumn{4}{|c|}{ Scorpion population } \\
\hline & \multicolumn{2}{|c|}{ Yunnan-sourced } & \multicolumn{2}{|c|}{ Hainan-sourced } \\
\hline & ESTs & clusters & ESTs & clusters \\
\hline $\mathrm{NaTx}$ & 43 & 12 & 53 & 16 \\
\hline$\alpha-K T x$ & 94 & 11 & 43 & 8 \\
\hline$\beta-\mathrm{KT} x$ & 12 & 4 & 24 & 2 \\
\hline LVPS & 69 & 7 & 10 & 6 \\
\hline calcine & 1 & 1 & 2 & 2 \\
\hline scamp & 26 & 2 & 30 & 2 \\
\hline plamp & 9 & 1 & 15 & 1 \\
\hline bpp like & 24 & 1 & 32 & 2 \\
\hline anionic peptide & 25 & 2 & 43 & 3 \\
\hline Glycine-rich peptide & 2 & 2 & 11 & 4 \\
\hline SPSV & 4 & 2 & 2 & 2 \\
\hline PLA 2 & 1 & 1 & 1 & 1 \\
\hline 8C-toxin & 4 & 2 & 3 & 3 \\
\hline salivary protein & 11 & 4 & 7 & 3 \\
\hline La1-like peptide & 3 & 2 & 2 & 1 \\
\hline Metalloprotease & 13 & 2 & 1 & 1 \\
\hline TIL peptide & 0 & 0 & 1 & 1 \\
\hline Cytotoxic peptide & 0 & 0 & 1 & 1 \\
\hline CITX & 0 & 0 & 1 & 1 \\
\hline Atypical possible toxin types & 62 & 19 & 44 & 23 \\
\hline Total Toxin-like peptides & 403 & 75 & 326 & 83 \\
\hline
\end{tabular}

We obtained $43 \mathrm{NaTxs}$ transcripts from the venom gland cDNA library of Yunnan-sourced Lychas mucronatus, accounting for nearly $11 \%$ of whole toxin-like peptide sequences. These sequences were grouped in 12 clusters. According to sequence similarity and targeting receptor site, NaTxs are assorted into two patterns: $\alpha-N a T x s$ and $\beta$-NaTxs [36]. 6 clusters putative $\alpha-N a T x s$ encoded by 11 ESTs were found in Yunnan-sourced population, whereas putative $\beta$-NaTxs possess of an obviously higher abundance which have 32 ESTs encoding 6 clusters.

One cluster of putative $\beta$-NaTxs (GT028621 and GT028626) encoded by 16 transcripts is the most abundant NaTx cluster in Yunnan-sourced population library. This cluster contains two almost identical sequences differing by only two amino acids. The transcript GT028621 encodes a mature peptide of 63 amino acid residues with 8 cysteines which would form a disulfide bridges pattern of "C-C-CX ${ }_{3} \mathrm{C}-\mathrm{C}-\mathrm{CXC}-\mathrm{C}$ " (C, cysteine; $\mathrm{X}$, amino acids of various types). This peptide shows homology to the beta-toxin Toxin CsEv1 identified from Centruroides sculpturatus. This homology may indicate that the most abundant 'long-chain' neurotoxin in the venom of Yunnan-sourced Lychas mucronatus can affect sodium channel activation by binding voltageindependently at site- 4 of sodium channels. Although the most abundant NaTx transcripts (EU159276 and EU159292) from Hainan-sourced library have the same cysteine pattern as GT028621, obvious diversity can be 
observed in primary structures. Specially, GT028621 don't contain a basic amino acid residue at $\mathrm{C}$-terminal region, which shows that $\mathrm{C}$-terminal modification would not exist at the post-translational level.

Although no significant difference was observed in the NaTx types from two scorpion populations of Lychas mucronatus, the NaTxs were distinct in primary structures (Figure 4). Moreover, the abundance of NaTxs was quite different. NaTxs account for nearly $16 \%$ of all toxin-like peptides in Hainan-sourced population, but $11 \%$ in Yunnan-sourced population. In addition, both scorpion populations possessed of a high abundant of putative $\beta$-NaTxs.

\section{$\alpha-K T x$ ( $\alpha$ subfamily of toxins specific for potassium channels)}

$\alpha$-KTx is widely spread in all scorpion species ever studied. This main toxin type possesses from 25 to 45 amino acid residues well packed with three or four disulfide bridges [31]. Their conserved three-dimensional structures are always constituted by a $\alpha$-helix connected to a two or three-stranded $\beta$-sheet [37].

In the scorpion Lychas mucronatus from Yunnan province, we obtained 11 clusters (94 ESTs) encoded for $\alpha-\mathrm{KTx}$. Among them, 9 clusters of putative $\alpha-\mathrm{KT}$ xs are constrained by 3 disulfide bridges, and the other 2 clusters are packed with four disulfide bridges (Figure 5).
GT028876 represents a 3 ESTs cluster encoding a putative $\alpha$-KTx. It has a mature peptide of 42 amino acid residues. Because of its unique primary structure, no homologies were shown after BLAST search. However, two clusters represented by EU163859 and EU163857 from Hainan-sourced population show some homology to GT028876. In respect that they have the same cysteine pattern as those function identified potassium channel toxins [25]. GT028876 may be a new group of short chain $\mathrm{K}^{+}$channel blockers.

GT028663 and GT028654 belong to the most abundant $\alpha-\mathrm{KTx}$ cluster which is encoded by 70 ESTs. Both transcripts were supposed to encode a mature peptide with 49 amino acid residues. The mature peptides are constrained by 4 disulfide bridges, which is distinct to most other putative $\alpha-\mathrm{KTx}$ toxins obtained from both scorpion populations. Blast search presented homologous Tx771 putative potassium channel toxin from Buthus occitanus Israelis and male-specific potassium channel inhibitor IsTX from Opisthacanthus madagascariensis which was proved to be a blocker to voltagegated potassium channels Kv1.1 and Kv1.3. Surprisingly, the highest abundant toxin type from Yunnan-sourced Lychas mucronatus has no homologous transcript in Hainan-sourced population. Although there is still no clue on exact function of this $\alpha-\mathrm{KTx}$ cluster, it is maybe

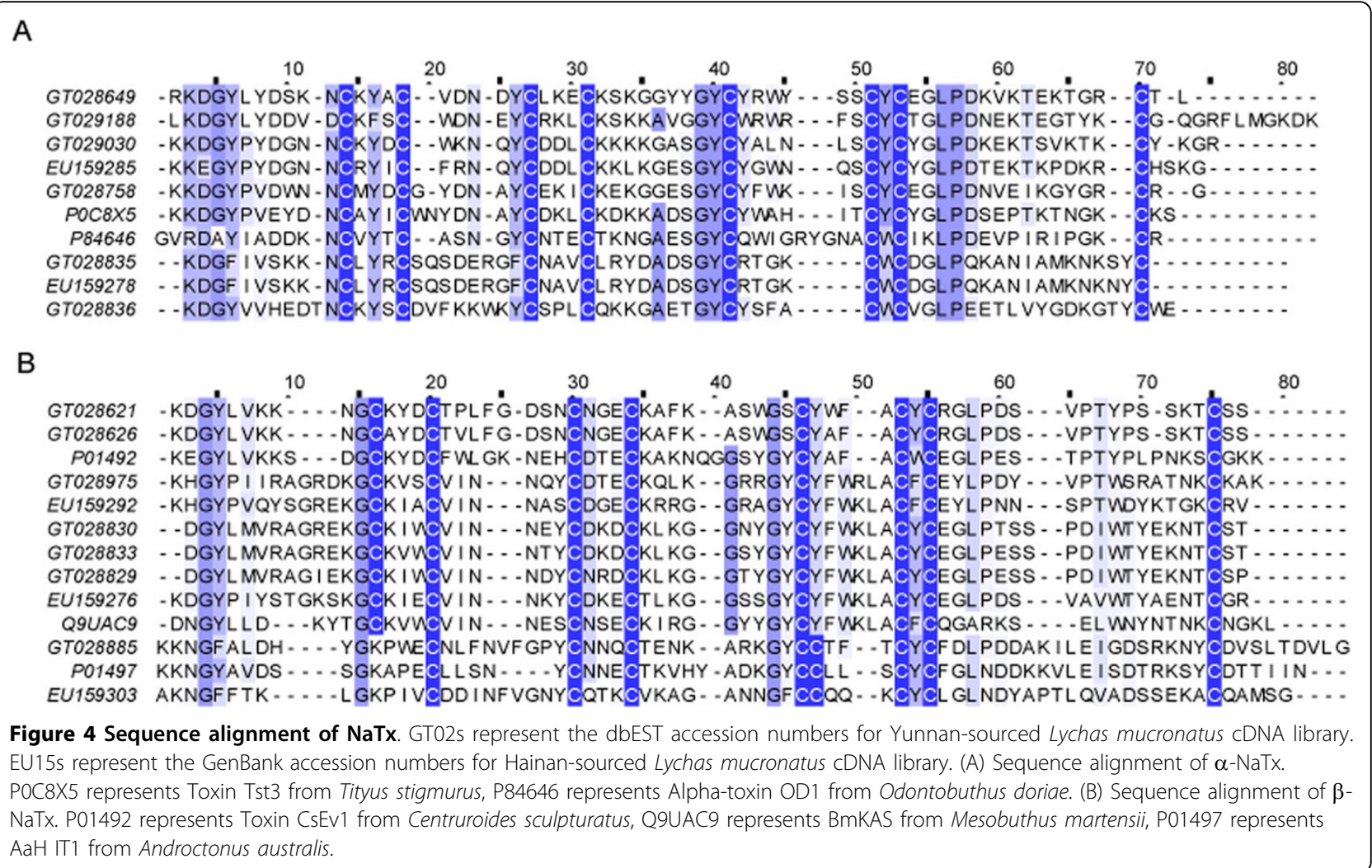




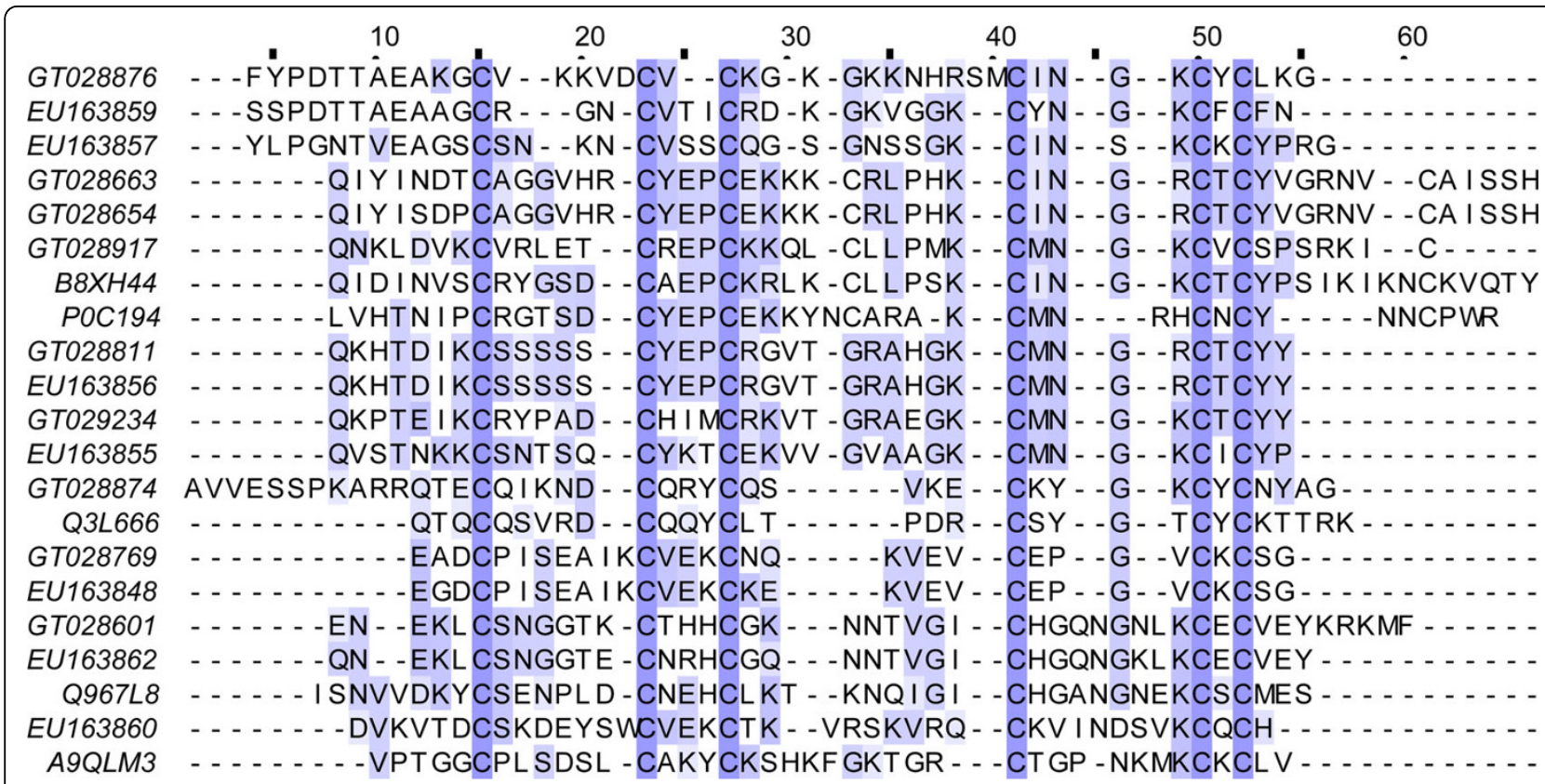

Figure 5 Sequence alignment of $\boldsymbol{\alpha}$-KTxs. GT02s represent the dbEST accession numbers for Yunnan-sourced Lychas mucronatus cDNA library EU15s represent the GenBank accession numbers for Hainan-sourced Lychas mucronatus CDNA library. A9QLM3 represents the first reported toxin (LmKTX8) from Hainan-sourced population. Q967L8 represents the defensin-like protein TXKS2 from Mesobuthus martensii. B8XH44 represents the putative potassium channel toxin Tx771 from Buthus occitanus Israelis. Q3L666 represents the KTx-like peptide kk4 from Mesobuthus martensii. POC194 represents the male-specific potassium channel inhibitor IsTX from Opisthacanthus madagascariensis.

very important for the survival of Yunnan-sourced Lychas mucronatus. Besides, another 2 ESTS cluster represented by GT028917 also possesses of the same cysteine pattern as above-mentioned sequences. These 72 ESTs form another new group of short chain $\mathrm{K}^{+}$ channel blockers together. EU163848 represents the most abundant $\alpha$-KTx cluster in Hainan-sourced population. It possesses of a 32 amino acid mature peptide with a pI of 4.82. This 31 ESTs encoded acidic $\alpha-K T x$ cluster has homologous cluster represented by GT028769 in Yunnan-sourced population. But the abundance of these acidic putative $\mathrm{K}^{+}$channel toxins is quite distinct between two populations and their functions are also to be identified.

Potassium scorpion toxins are usually classified into 4 subfamilies: $\alpha, \beta, \gamma$ and $\kappa$ KTxs [13]. Each subfamily consists of several groups. Based on similar analysis as described before [38], GT028811 and GT029234 belong to $\alpha-\mathrm{KTx} 12$ group, GT028874 belongs to $\alpha-\mathrm{KTx} 17$ group, and the other short chain KTxs constitute at least 4 new groups. $\alpha-\mathrm{KTx}$ is the most abundant toxin type in Yunnan-sourced population. It takes parts of nearly $23 \%$ of total toxin-like peptides, which is nearly 2 -fold higher than $13 \%$ in Hainan-sourced population. In a word, not only the primary structures, but also the abundance of $\alpha-K T x s$ between two scorpion populations displayed high diversity.

\section{Other neurotoxins}

There are two other neurotoxin types identified from Yunnan-sourced Lychas mucronatus. One type belongs to $\beta-\mathrm{KTx}$, which is previously called "orphan peptides". This type is constituted by a freely moving amino acid sequence at $\mathrm{N}$-terminal region and a cysteine-stabilized $\alpha \beta$ motif at C-terminal region [39]. We obtained 4 clusters (12 ESTs) encoding $\beta$-KTx from Yunnan-sourced Lychas mucronatus (Figure 6). The primary structures of these long-chain $\mathrm{K}^{+}$channel toxins show high homology between two scorpion populations. It was worth mentioning that two transcripts GT028643 and GT028645 with only 3 amino acid residues difference in N-terminal region were proved to be encoded by a single gene and generated by alternative 3' splice sites. The results of electrophysiological experiments showed that the recombinant protein of these two transcripts have a very weak effect to block voltage-gated potassium channels Kv1.1 which means the real target of these $\beta$-KTxs is still waiting to be identified (Data preparing for publish). They also represent the most abundant cluster of $\beta-\mathrm{KTx}$ in the venom of Yunnan-sourced Lychas mucronatus. Based on similar analysis as described before $[13,38]$, GT028816 is a new member of $\beta-K T x 1$ group, while GT028643, GT028645 and GT028908 are new members of $\beta-\mathrm{KTx} 2$ group. All the ESTs encoded for $\beta-\mathrm{KTx}$ in Yunnan-sourced population take parts of $3 \%$ of whole 


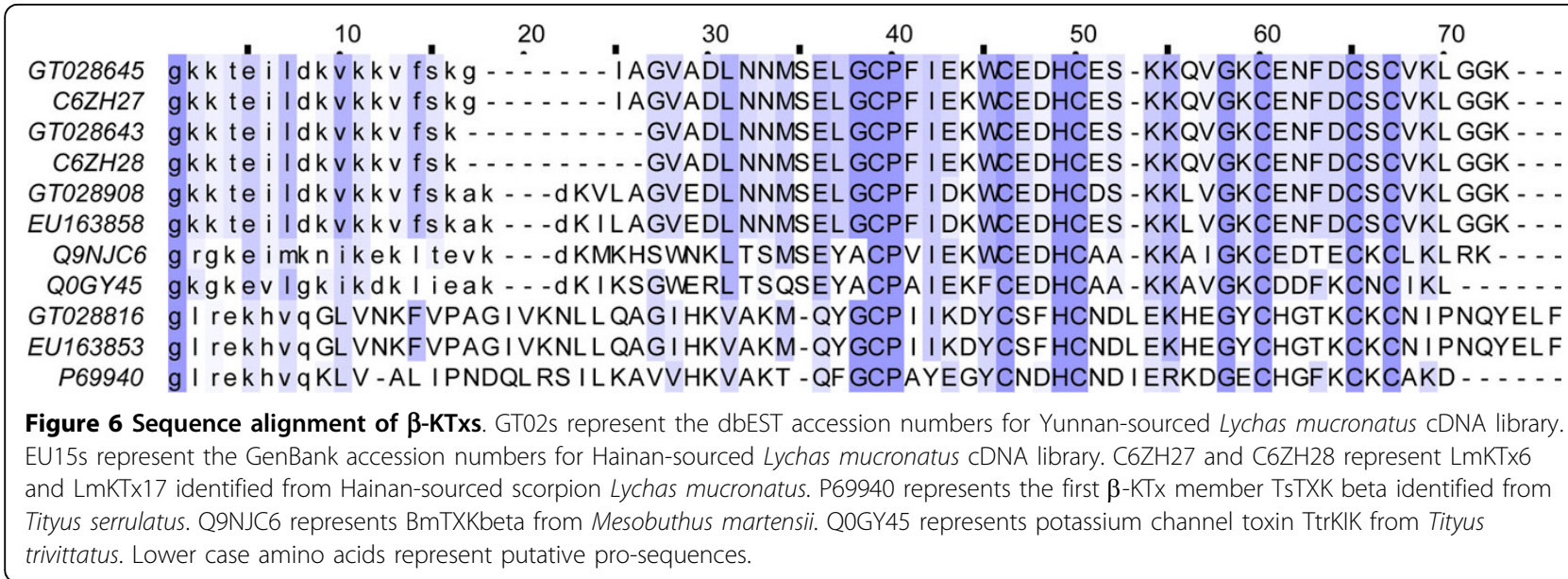

toxin-like peptides which is obviously less than the $7 \%$ in Hainan-sourced population.

Till now, fewer $\mathrm{Ca}^{2+}$ channel toxins have been obtained from scorpion venoms [40]. This type of toxin specific for ryanodine receptors possesses of an ICK (inhibitory cysteine knot) motif at C-terminal region. One EST GT029194 encoding calcine was identified from the library of Yunnan-sourced Lychas mucronatus. GT029194 has an 8 amino acid residues long pro-peptide and a mature peptide of 34 amino acid residues constrained by three disulfide bridges. FE193690 from Hainan-sourced population has the same primary structure as GT029194, which suggests that the calcine is conserved between two scorpion populations (Figure 7). Both of the transcripts show the conserved cysteine pattern like Tx758 from Buthus occitanus Israelis and Opicalcine-1 from Opistophthalmus carinatus. So that, GT029194 should be a new member of calcium channel toxins group 1 based on similar analysis as described before [13,38].

AMPs (antimicrobial peptides)

AMPs are a wide class of venom peptides with antimicrobial functions [8]. It is an important defensive weapon of innate immunity for scorpion [41]. AMPs take on great diversity in primary amino acid sequences.
Most of them are short cationic peptides [42], and are structurally divided into three groups, namely linear peptides with an amphipathic $\alpha$-helix, cysteine-rich peptides with one or several disulfide bridges [43], and peptides with a predominance of specific amino acids such as glycine, proline and histidine [44]. In a word, AMPs form the first line of host defense against pathogenic infections.

In Yunnan-sourced Lychas mucronatus, 5 type peptides are presumed to belong to AMPs: Short cationic antimicrobial peptides (Scamp), Ponericin-like antimicrobial peptides (Plamp), bradykinin-potentiating peptide like (bpp like), Glycine-rich peptides and Anionic peptides.

Scamp is a type of low molecular weight peptides belonging to the family of cationic host defense peptides [45]. This toxic type shows homology to small linear cationic toxins BmKb1 identified from the scorpion Mesobuthus martensii [46]. This kind of peptide always possesses of a conserved proline in the seventh amino acid residue and a lysine in the $\mathrm{C}$-terminal region of mature peptide [24]. Our group had already identified one Scamp mucroporin from the Hainan-sourced Lychas mucronatus, which can effectively inhibit standard bacteria, especially gram-positive bacteria.

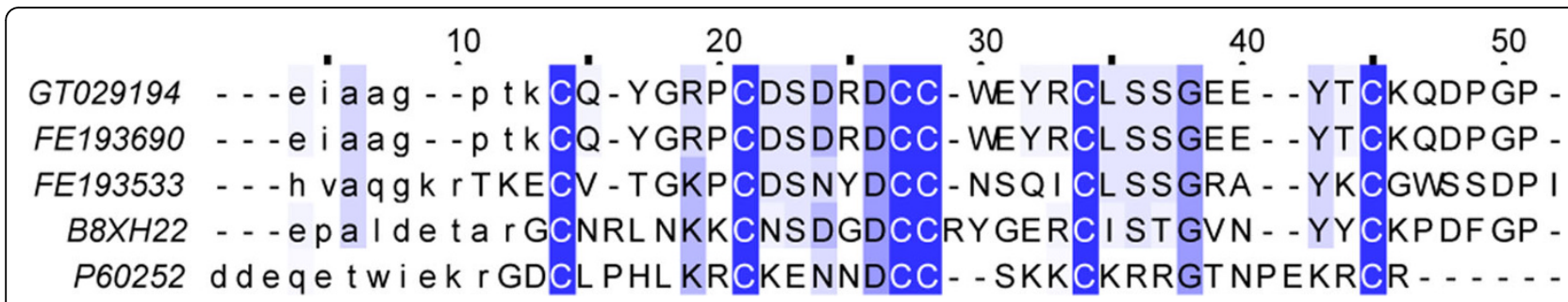

Figure 7 Sequence alignment of Calcine. GT02s represent the dbEST accession numbers for Yunnan-sourced Lychas mucronatus cDNA library. FE19s represent the dbEST accession numbers for Hainan-sourced Lychas mucronatus CDNA library. B8XH22 represents Tx758 from Buthus occitanus Israelis. P60252 represents Opicalcine-1 from Opistophthalmus carinatus. Lower case amino acids represent putative pro-sequences. 


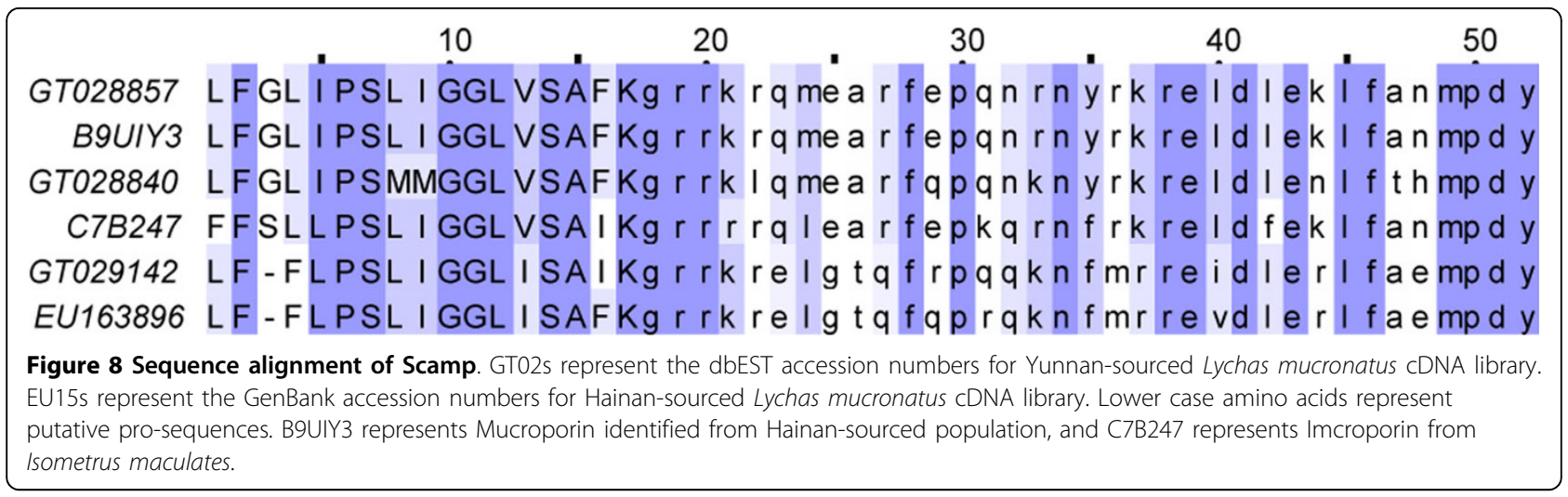

From Yunnan-sourced Lychas mucronatus, we obtained 2 clusters (26 ESTs) of Scamp. Among them, GT028857 shared the same primary structure as mucroporin. Actually, both two clusters of Scamp obtained from Yunnansourced Lychas mucronatus have homologous transcripts in Hainan-sourced population venom (Figure 8). Scamp possesses of a high abundance in both scorpion populations venom (more than 6\% in Yunnan-sourced population and $9 \%$ in Hainan-sourced population).

From Yunnan-sourced Lychas mucronatus, we obtained 1 clusters (9 ESTs) encoded for Plamp, which was a homologous toxin type of Ponericins identified from ant venom with a high activeness against Grampositive and Gram-negative bacteria [47]. Plamp has a mature peptide consist of 24-25 amino acid residues, most of which are Lys and Arg. The primary structures of Plamps between two scorpion population are greatly conserved (Figure 9), but its abundance in Yunnansourced population is obviously lower than Hainansourced population.

Until now, bpp like are only characterized from the venom of family Buthidae. This type of toxin is represented by Bradykinin-potentiating peptide BmK3 identified from the scorpion Mesobuthus martensii [48]. From Yunnan-sourced Lychas mucronatus, we obtained 1 clusters (24 ESTs) encoded for this toxin type, represented by GT028786. GT028786 has almost identical primary structure like EU163895 from Hainan-sourced population (Figure 10). According to sequence alignment analysis, the N-terminal region of GT028786 shows homology to parabutoporin which has a strong antibacterial activity against Gram-negative bacteria and fungal [49]. As a result, the special primary structure of scorpion bpp like with one putative antimicrobial region at $\mathrm{N}$-terminus

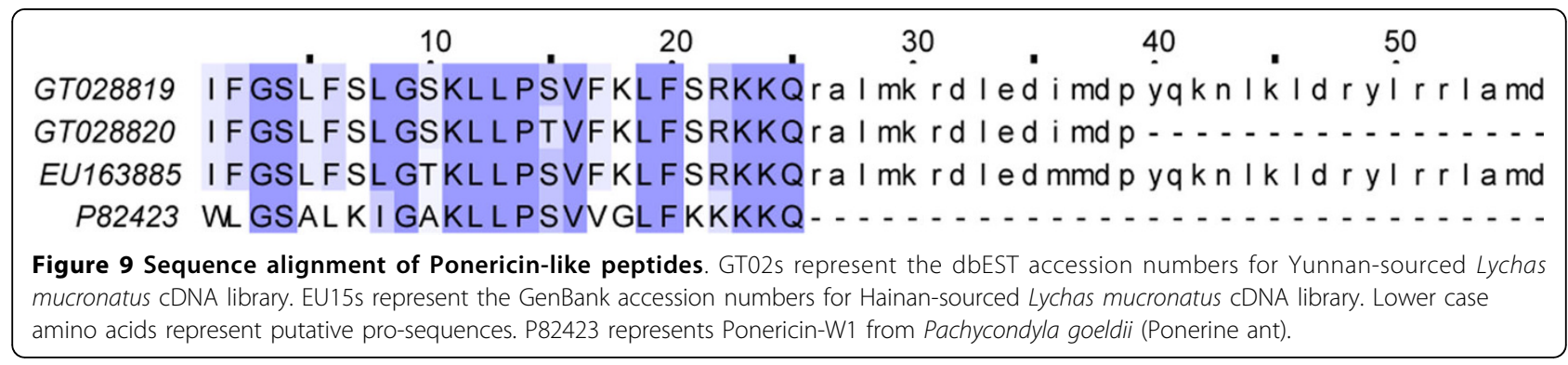

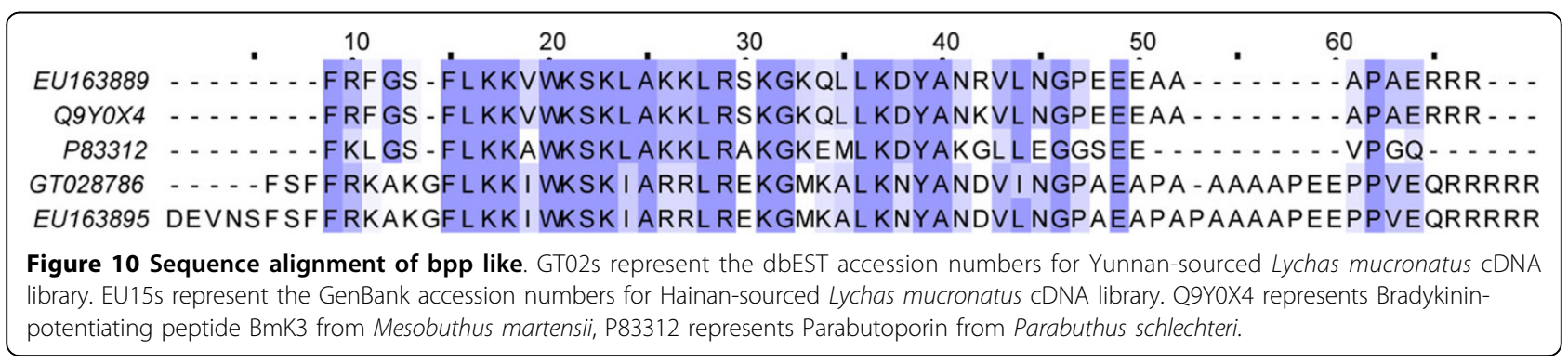




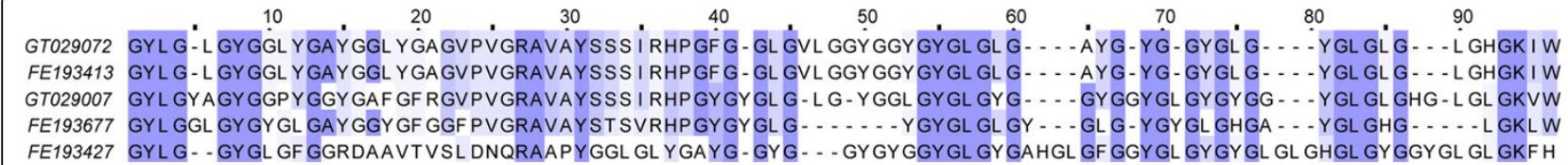

Figure 11 Sequence alignment of Glycine-rich peptides. GT02s represent the dbEST accession numbers for Yunnan-SOurced Lychas mucronatus cDNA library. FE19s represent the dbEST accession numbers for Hainan-sourced Lychas mucronatus cDNA library.

and the other putative Bradykinin-potentiating region at C-terminus makes it to be a hypothetic bi-function protein.

Glycine-rich peptides are a type of specific amino acid predominant peptides first characterized from scorpion by our group. In this work, 2 ESTs encoded for Glycinerich peptides were obtained from Yunnan-sourced population (Figure 11). After a multiple sequence alignment analysis, we could find that Glycine-rich peptides in Hainan-sourced population have more diversity and also a higher abundance.

Anionic peptides are a type of acidic venom peptides rich in aspartic acid and glutamic acid residues. Such toxin type was previously obtained from both the family Buthidae and non-Buthidae [30,50]. To the best of our knowledge, this acidic toxin type takes a higher abundance in venom of family Buthidae than non-Buthidae. Considering the family Buthidae possesses a higher abundance of basic neurotoxins in venom. It may imply that Anionic peptides play an important role in balancing the $\mathrm{pH}$ value of scorpion venom liquid [30]. 2 clusters (25 ESTs) encoded for Anionic peptides were obtained from Yunnan-sourced population. Both clusters represented by GT028615 and GT028723 have homologous transcripts in Hainan-sourced population (Figure 12). In Hainan-sourced population, anionic peptides account for $12 \%$ of total toxin-like peptides, which is 2 -fold higher than $6 \%$ in Yunnan-sourced population.

Although there was still no consensus about the role of AMPs in scorpion venom, they were postulated to have the functions of protecting the scorpion from bacterial infection, depolarizing neural cells inducing immobilization of prey and potentiating the action of other neurotoxins within the venom [51]. 8 clusters (86 ESTs) encode for AMPs were obtained from Yunnansourced Lychas mucronatus, which accounted for $21 \%$ of all toxin-like peptides. However, the abundance of AMPs reached remarkable $40 \%$ in Hainan-sourced Lychas mucronatus. The primary structure of AMPs from Hainan-sourced Lychas mucronatus showed more diversity. This comparison suggested that Hainansourced Lychas mucronatus definitely can protect itself more effectively from microorganisms.

\section{Other venom components}

These venom components include 7 known toxin types and nineteen atypical types of venom molecules. The 7 known toxin types are lipolysis activating peptides (LVPs), phospholipase A2 (PLA2), serine protease from scorpion venom (SPSV), metalloprotease, salivary protein, La1-like peptides and $8 \mathrm{C}$ toxin. LVPs are a group of long chain toxins shared high homology to those NaTxs but in defect of a cysteine. This structure makes LVPs form an interchain disulfide bridge and exert distinct biological activity on adipocyte lipolysis [52]. 7 clusters (69 ESTs) encoding LVPs were identified from Yunnansourced Lychas mucronatus. It was worth mentioning that LVPs were the second most abundant toxin type in the venom of Yunnan-sourced population. It accounted for $17 \%$ of whole toxin-like peptides far more than $3 \%$ in Hainan-sourced population. Although the abundance of LVPs was quite distinct in two populations, their primary structures seemed conserved (Figure 13). PLA2, SPSV and metalloprotease are proteinases within scorpion venom. Their abundances are correspondingly lower 


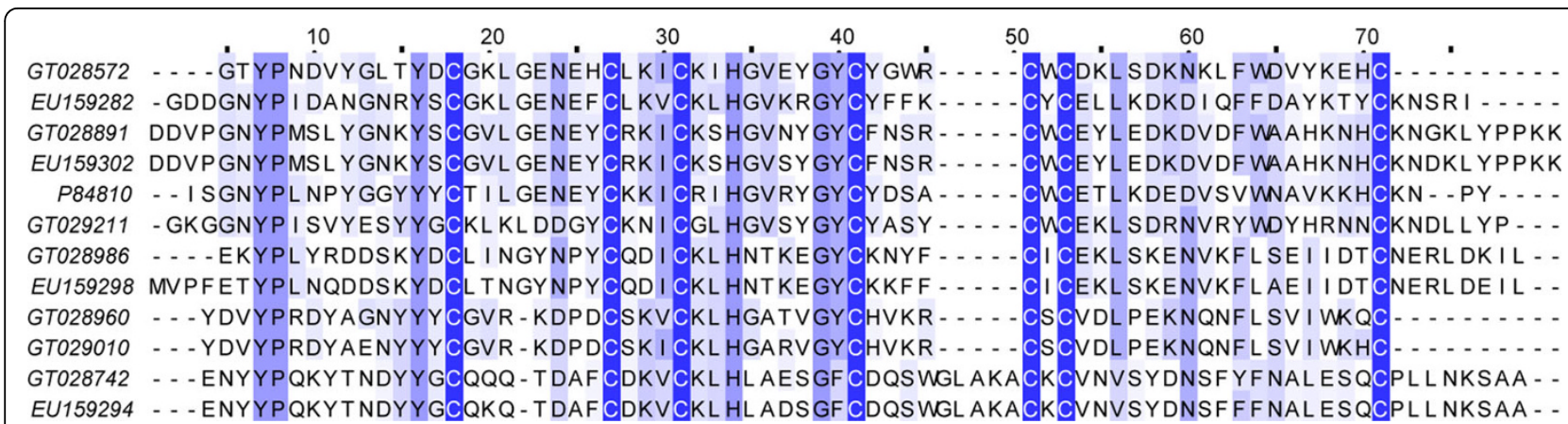

Figure 13 Sequence alignment of LVPs. GT02s represent the dbEST accession numbers for Yunnan-sourced Lychas mucronatus cDNA library. EU15s represent the GenBank accession numbers for Hainan-sourced Lychas mucronatus cDNA library. P84810 represents Lipolysis-activating peptide 1-alpha chain from Buthus occitanus tunetanus.

than those "common" toxin types in venom, but without a doubt, they are very important for scorpion to prey and digest. Salivary protein is a type of high molecular weight protein in scorpion venom. Salivary protein was denominated for its homology to the salivary derived protein from tick, and this type of toxins always has a mature peptide of about 210 amino acid residues. La1 is the most abundant toxin identified from the venom of Liocheles australasiae [9]. But the abundance of La1-like peptides in venom of family Buthidae is not such high, and the accurate function of La1-like peptides is still unknown. $8 \mathrm{C}$ toxin is another new type of toxin-like peptides well packed by 4 disulfide bridges, which was first obtained by our group. Nineteen atypical types of venom molecules obtained from Yunnan-sourced population were seldom reported in other scorpion venoms. The accurate function of these atypical scorpion venom toxins is still needed to be characterized [see Additional file 1]. Other venom components accounted for nearly $41 \%$ of whole toxin-like peptides in venom of Yunnansourced population, which is a big natural venom resource waiting for exploring.

\section{Discussion}

Scorpion venom is a complex mixture of biologically active peptides with diverse physiological effects [14]. Most of the peptides ever identified are disulfide-rich neurotoxins that specifically modulate various ion channels permeability of excitable and non-excitable cells [31]. However, more and more other venom components have been characterized recently, including antimicrobial peptides without disulfide bridges and other functional molecules [32,53]. In order to gain further insight into scorpion venom compositions, it is not enough to employ routine studies by protein chemistry always aiming at the isolation of specific active components. The introduction of powerful chromatographic techniques, followed by primary structure determination using automatic Edman degradation, makes it possible to produce an overview of scorpion venom compositions. Mass spectrometry was firstly used to get the mass fingerprinting of toxic fractions of Tityus serrulatus venom [54]. Since then, overall venom compositions of ten scorpion species have been comprehensively investigated at the proteome level, including eight species from the family Buthidae [14]. The powerful technique help to confirm the hypothesis that scorpion venom is a complex mixture of various distinct proteins with vastly abundant difference between families to families, genus to genus and different species within a genus [9]. We chose transcriptomic approach to get an overview of the Yunnan-sourced Lychas mucronatus venom because it's not affected by the extraction of venom and the dynamic expression of the gland or peptide maturation like proteomic approach [12]. The transcriptomic analysis is more likely to give a comprehensive comparison of the venom compositions from two Lychas mucronatus populations.

Some evidences already displayed the phenomenon of intraspecific diversity of scorpion venom peptides $[12,26]$. According to SDS-PAGE and random amplified polymorphic DNA (RAPD) techniques, the venom of Scorpio maurus palmatus from four geographically isolated localities in Egypt was investigated. Both obvious morphological differences and protein molecular weights diversity were observed [55]. But the particular distinctions such as primary structure and exact abundance of venom toxins among different populations of the same scorpion species are not clearly clarified. As two populations of the scorpion Lychas mucronatus successively obtained from Hainan and Yunnan provinces were convinced to be the same species according to morphological analysis, our study conduct on the venom components of Lychas mucronatus by a transcriptome approach may fitly figure out the question. 
The experimental methods for constructing the venom gland cDNA library of Yunnan-sourced Lychas mucronatus was the same as Hainan-sourced Lychas mucronatus [23]. 60 adult specimens from both scorpion populations were selected when venom gland cDNA library was constructed. The gender difference of scorpions is basically half to half. We cut off scorpion venom glands 2 days after extraction of their venom by electrical stimulation. Most temporary influences such as age, seasonal, sex, feeding behavior and time for RNA transcription can be excluded. Nevertheless, the experimental results confirmed our hypothesis about the diversity exist in nearly every toxin type (Table 2).

Almost all high-abundant toxin types were obtained from two scorpion populations. Great diversity was observed in these highly expressed venom compositions, especially neurotoxins and AMPs. Among neurotoxins, short chain $\alpha$-KTx was highly expressed in Yunnansourced population, whereas long chain NaTx and $\beta$ KTx was highly expressed in Hainan-sourced population. Particularly, $\alpha-\mathrm{KTx}$ in the venom of Yunnansourced population is not only 2 -fold higher abundant than in Hainan-sourced population, but also more diversified in primary structures. GT028663 represents the most abundant $\alpha-\mathrm{KTx}$ cluster in Yunnan-sourced population, but we can not find its homologous transcripts in the venom gland cDNA library of Hainan-sourced population. EU163848 represents the most abundant $\alpha-\mathrm{KTx}$ cluster from Hainan-sourced population. Although EU163848 shows homology to GT028601 from Yunnansourced population, EU163848 obviously has a higher expression level. Taken together, Yunnan-sourced Lychas mucronatus possesses a higher abundant and more diversified $\alpha$-KTxs in venom and the homologous transcripts of the most abundant $\alpha-K T x$ cluster in the venom of Hainan-sourced Lychas mucronatus have a relatively low expressed level in the venom of Yunnansourced Lychas mucronatus. All these obvious diversity should be intricately related to scorpions' adaption in different environment which primarily concern the scorpions' interaction with their prey and predators. On the other hand, Hainan-sourced Lychas mucronatus possesses a higher abundance of NaTxs in venom. Because the NaTxs can cause severe neurotoxic symptoms after scorpion envenomation [36], Hainan-sourced Lychas mucronatus could be a more dangerous killer, which is more efficient for not only preying but also defensing itself from other predators.

According to the transcriptome analysis, we can conclude that both scorpion populations possess relatively high abundant AMPs. But it's unexpected that the abundance of AMPs in the venom of Hainan-sourced population reached remarkable $40 \%$ of all toxin-like peptides. Till now, more and more AMPs have been isolated and characterized [56,57]. AMPs can destroy bacteria, fungi, parasites, and even some viruses [58]. They are important defensive weapons of scorpion innate immunity. So we can assume that the high expression levels of AMPs could protect Hainan-sourced Lychas mucronatus away from microbial infection, and it may also implicate other unascertained benefits for the survival of the population.

Hainan island locates in Southern China and is separated from mainland by Qiongzhou strait. Because surrounded by South China Sea, the climate of Hainan island is hot and humid all the year round. Yunnan locates in southwest China and is a mountainous region, whose rainfall is relatively less than Hainan province. Hainan island is an excellent living environment for Lychas mucronatus. In this biotope, Hainan-sourced Lychas mucronatus has enough prey. But this hot and humid environment may also bring powerful nature enemy and more pathogenicbacteria. In a word, the distinctive living environments probably relate to the adaptive evolution of two Lychas mucronatus populations.

The dissimilarity of toxin-like peptides between two populations was showed in Figure 14. Although two populations of scorpion belong to the same species, diversity of venom peptides had gradually accumulated since Hainan island was separated from Chinese mainland 2.5 million years ago [59]. Long period isolation doesn't make the scorpion Lychas mucronatus change their toxin types much in venom, but the primary structure and abundance of these toxin types change a lot which probably related to the adaption of Lychas mucronatus in different environment. To the best of our knowledge, this work is the first comprehensive comparative analysis of venom transcriptomes between two populations of the same scorpion species from different geographical regions. It provides accurate difference of venom peptides between Lychas mucronatus from Hainan and Yunnan. Sight is no longer restricted to only protein molecular weights and concentration, but also the detailed diversity of primary structure and abundance. Besides, because two scorpion populations are obtained successively from island and mainland, Lychas mucronatus could represents a useful model system for studying molecular evolution and biogeography. In addition, the geographical variability in the venom of the same species also provides some clues for producing of effective anti-venoms and understanding the symptomatology of envenomation.

\section{Conclusions}

Our work unravels a large number of venom molecules never identified in scorpion species. The result provides a comprehensive comparative analysis of venom transcriptomes of the same scorpion species from different 


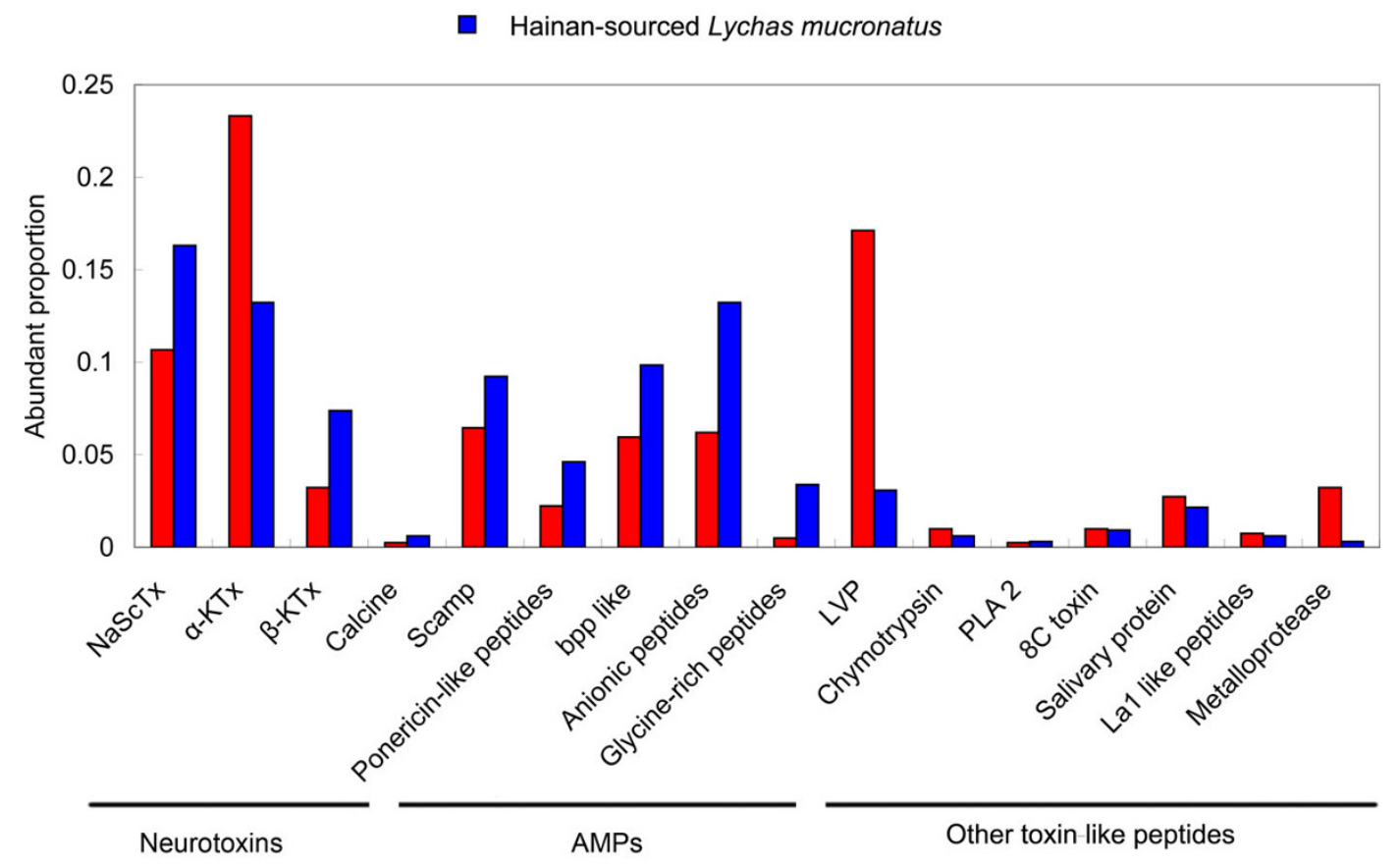

Toxin types

Figure 14 Comparison of abundance of different toxin types between two populations of Lychas mucronatus. For instance, there are 94 ESTs encoded for $\alpha$-KTX in the library of Yunnan-sourced Lychas mucronatus accounting for $24 \%$ of all the 396 ESTs encode for toxin-like peptides.

geographical regions, which thoroughly reveals the fact that peptides and proteins of the same scorpion species from different geographical regions are highly diversified and the scorpion venom arsenal is a constantly evolving system to adapt the different biotopes. We can make a conclusion that there are far more peptides in one scorpion species venom than previous expectation considering of the geographical isolation.

\section{Methods}

\section{cDNA library construction}

The Yunnan-sourced Lychas mucronatus were collected from Shidian county of Yunnan province in September 2008. Venom glands of 60 wild specimens were cut off 2 days after extraction of their venom by electrical stimulation [60], and ground into fine powder in liquid nitrogen. Total RNA was isolated with TRIZOL Reagent (Invitrogen, Carlsbad, CA, USA), and then mRNA was prepared with PolyATtract ${ }^{\circ}$ mRNA Isolation Systems (Promega, Madison, WI, USA). SuperScript ${ }^{\text {tw }}$ Plasmid System (Invitrogen) was used to construct a directional cDNA library from $6 \mu \mathrm{g}$ mRNA. cDNA inserts were directionally cloned into the plasmids PSPORT 1 according to the supplier's instructions. The recombinant plasmids were transformed into electrocompenent cells.

\section{Sequencing}

After growing the clones overnight in appropriate Luria Broth culture medium containing $100 \mu \mathrm{g} / \mathrm{ml}$ of ampicillin, random colonies were selected in order to obtain an unbiased overview of the venom gland transcriptome. After overnight culture, plasmid DNA was isolated using alkaline lysis method. Purified plasmids were single-pass sequenced on ABI 3730 automated sequencers (Applied Biosystems, Foster City, CA, USA).

\section{Bioinformatics analysis}

Phred program were used to examine the trace files of sequenced clones, the cutoff Phred score was set to 40 as before $[30,61]$. Vector and adaptor sequences were removed using the program Cross Match. After removing the PolyA tail, we discarded those sequences shorter than 100 nt. High-quality sequences were processed on the website EGassembler http://egassembler.hgc.jp/ with the default parameter [28]. The resulted sequences were deposited into the dbEST. Each cluster was annotated 
by being searched against SWISS-PROT http://www. expasy.org/tools/blast/ and GenBank NCBI database http://www.ncbi.nlm.nih.gov/blast using BLAST algorithms with an e-value cut-off set to $<10^{-4}$. After BLAST search, the unmatched clusters were further identified for open reading frames using the ORFfinder http://www.ncbi.nlm.nih.gov/projects/gorf/. Considering the extreme diversity of scorpion toxins, those clusters putative to encode venom peptides was re-examined manually to pick out individual different isoforms. All clusters were checked for the existence of signal peptides using the SignalP 3.0 program http://www.cbs.dtu. $\mathrm{dk} /$ services/SignalP/ [62]. All the sequence alignment was performed by Clustal_X 1.83 software followed by manual adjustment [63], and viewed by the software Jalview [64].

\section{Additional material}

Additional file 1: Atypical venom molecules characterized from the scorpion Yunnan-sourced Lychas mucronatus. The data represents nineteen novel types of venom peptides with special primary structures and unrevealed functions from the scorpion Yunnan-sourced Lychas mucronatus.

\section{Acknowledgements}

This work was supported by grants from the Basic Project of Ministry of Science and Technology of China (No. 2007FY210800) and the Major State Basic Research Development Program of China (Nos. 2005CB522903, 2010CB529800, 2010CB530100), the China Specific Project for Developing New Drugs (Nos. 2009ZX09103-612 and 2008ZX10001-015-9), and the Program for Changjiang Scholars and Innovative Research Team in University (No. IRT0745).

\section{Authors' contributions}

Ruiming Zhao carried out cDNA library construction, participated in the bioinformatics analysis, and drafted the manuscript. Yibao Ma participated in the alignment analysis, and drafted the manuscript. Yawen He participated in the sequencing. Zhiyong Di completed the characterization of scorpion species. Yingliang Wu participated in the design and coordination of the study. Zhijian Cao participated in the design and coordination of the study, and drafted the manuscript. Wenxin Li conceived of the study, and participated in its coordination. All authors have read and approved the final manuscript.

Received: 6 May 2010 Accepted: 28 July 2010 Published: 28 July 2010

\section{References}

1. David WS: The Biology of Scorpions. Stanford: Stanford University PressGary, A. Polis 1990.

2. Michael ES, Victor F: High-level systematics and phylogeny of the extant scorpions (Scorpiones: Orthosterni). Euscorpius 2003, 11:1-175.

3. Possani LD, Becerril B, Delepierre M, Tytgat J: Scorpion toxins specific for Na+-channels. Eur J Biochem 1999, 264(2):287-300.

4. Schwartz EF, Diego-Garcia E, Rodriguez de la Vega RC, Possani LD: Transcriptome analysis of the venom gland of the Mexican scorpion Hadrurus gertschi (Arachnida: Scorpiones). BMC Genomics 2007, 8:119.

5. Rates B, Ferraz KK, Borges MH, Richardson M, De Lima ME, Pimenta AM: Tityus serrulatus venom peptidomics: assessing venom peptide diversity. Toxicon 2008, 52(5):611-618.

6. Catterall WA: Neurotoxins that act on voltage-sensitive sodium channels in excitable membranes. Annu Rev Pharmacol Toxicol 1980, 20:15-43.
7. Miller C, Moczydlowski E, Latorre R, Phillips M: Charybdotoxin, a protein inhibitor of single $\mathrm{Ca} 2+$-activated $\mathrm{K}+$ channels from mammalian skeletal muscle. Nature 1985, 313(6000):316-318.

8. Zasloff M: Antimicrobial peptides of multicellular organisms. Nature 2002, 415(6870):389-395.

9. Miyashita M, Otsuki J, Hanai Y, Nakagawa Y, Miyagawa H: Characterization of peptide components in the venom of the scorpion Liocheles australasiae (Hemiscorpiidae). Toxicon 2007, 50(3):428-437.

10. Batista CV, Roman-Gonzalez SA, Salas-Castillo SP, Zamudio FZ, GomezLagunas F, Possani LD: Proteomic analysis of the venom from the scorpion Tityus stigmurus: biochemical and physiological comparison with other Tityus species. Comp Biochem Physiol C Toxicol Pharmacol 2007, 146(1-2):147-157.

11. Dyason K, Brandt W, Prendini L, Verdonck F, Tytgat J, du Plessis J, Muller G, van der Walt J: Determination of species-specific components in the venom of Parabuthus scorpions from southern Africa using matrixassisted laser desorption time-of-flight mass spectrometry. Rapid Commun Mass Spectrom 2002, 16(8):768-773.

12. Pimenta AM, De Marco Almeida F, de Lima ME, Martin-Eauclaire MF, Bougis PE: Individual variability in Tityus serrulatus (Scorpiones, Buthidae) venom elicited by matrix-assisted laser desorption/ionization time-of-flight mass spectrometry. Rapid Commun Mass Spectrom 2003, 17(5):413-418.

13. Tan PT, Veeramani A, Srinivasan KN, Ranganathan S, Brusic V: SCORPION2: a database for structure-function analysis of scorpion toxins. Toxicon 2006, 47(3):356-363.

14. Rodriguez de la Vega RC, Schwartz EF, Possani LD: Mining on scorpion venom biodiversity. Toxicon 2009.

15. Oukkache N, Rosso JP, Alami M, Ghalim N, Saile R, Hassar M, Bougis PE, Martin-Eauclaire MF: New analysis of the toxic compounds from the Androctonus mauretanicus mauretanicus scorpion venom. Toxicon 2008, 51(5):835-852.

16. Kozminsky-Atias A, Bar-Shalom A, Mishmar D, Zilberberg N: Assembling an arsenal, the scorpion way. BMC Evol Biol 2008, 8:333.

17. Mao X, Cao Z, Yin S, Ma Y, Wu Y, Li W: Cloning and characterization of BmK86, a novel K+ -channel blocker from scorpion venom. Biochem Biophys Res Commun 2007, 360(4):728-734.

18. Remijsen Q, Verdonck F, Willems J: Parabutoporin, a cationic amphipathic peptide from scorpion venom: Much more than an antibiotic. Toxicon 2009.

19. Corzo G, Papp F, Varga Z, Barraza O, Espino-Solis PG, Rodriguez de la Vega RC, Gaspar R, Panyi G, Possani LD: A selective blocker of Kv1.2 and Kv1.3 potassium channels from the venom of the scorpion Centruroides suffusus suffusus. Biochem Pharmacol 2008, 76(9):1142-1154.

20. D'Suze G, Schwartz EF, Garcia-Gomez BI, Sevcik C, Possani LD: Molecular cloning and nucleotide sequence analysis of genes from a CDNA library of the scorpion Tityus discrepans. Biochimie 2009, 91(8):1010-1019.

21. Kovařik F: Revision of the genera Lychas and Hemilychas, with descriptions of six new species (Scorpiones: Buthidae). Acta Societatis Zoologicae Bohemicae 1997, 61:311-371.

22. Zhu M, Qi J, Song D: A Checklist of Scorpions from China (Arachnida: Scorpiones). Acta Arachnologica Sinica 2004, 13(2):111-118.

23. Liu J, Ma Y, Yin S, Zhao R, Fan S, Hu Y, Wu Y, Cao Z, Li W: Molecular cloning and functional identification of a new $\mathrm{K}(+)$ channel blocker, LmKTx10, from the scorpion Lychas mucronatus. Peptides 2009, 30(4):675-680

24. Dai C, Ma Y, Zhao Z, Zhao R, Wang Q, Wu Y, Cao Z, Li W: Mucroporin, the first cationic host defense peptide from the venom of Lychas mucronatus. Antimicrob Agents Chemother 2008, 52(11):3967-3972.

25. Wu W, Yin S, Ma Y, Wu YL, Zhao R, Gan G, Ding J, Cao Z, Li W: Molecular cloning and electrophysiological studies on the first $\mathrm{K}(+)$ channel toxin (LmKTx8) derived from scorpion Lychas mucronatus. Peptides 2007, 28(12):2306-2312.

26. Smertenko A, Omran MAA, Hussey PJ, McVean A: Toxin evolution in scorpion venom: Evidence for toxin divergence under strong negative selection in leiurus quinquestriatus subspecies. Toxin Reviews 2001, 20(3\&4):229-244

27. Gantenbein B, Largiader CR: The phylogeographic importance of the Strait of Gibraltar as a gene flow barrier in terrestrial arthropods: a case study with the scorpion Buthus occitanus as model organism. Mol Phylogenet Evol 2003, 28(1):119-130. 
28. Masoudi-Nejad A, Tonomura K, Kawashima S, Moriya Y, Suzuki M, Itoh M, Kanehisa M, Endo T, Goto S: EGassembler: online bioinformatics service for large-scale processing, clustering and assembling ESTs and genomic DNA fragments. Nucleic Acids Res 2006, , 34 Web Server: W459-462.

29. Yang Y, Cun S, Xie X, Lin J, Wei J, Yang W, Mou C, Yu C, Ye L, Lu Y, et al: EST analysis of gene expression in the tentacle of Cyanea capillata. FEBS Lett 2003, 538(1-3):183-191.

30. Ma Y, Zhao R, He Y, Li S, Liu J, Wu Y, Cao Z, Li W: Transcriptome analysis of the venom gland of the scorpion Scorpiops jendeki: implication for the evolution of the scorpion venom arsenal. BMC Genomics 2009, 10:290.

31. Rodriguez de la Vega RC, Possani LD: Current views on scorpion toxins specific for K+-channels. Toxicon 2004, 43(8):865-875.

32. Zhao Z, Ma Y, Dai C, Zhao R, Li S, Wu Y, Cao Z, Li W: Imcroporin, a new cationic antimicrobial peptide from the venom of the scorpion Isometrus maculates. Antimicrob Agents Chemother 2009, 53(8):3472-3477.

33. Ma Y, Zhao R, Li S, Fan S, Wu Y, Liu H, Cao Z, Li W: Characterization of LmTxLP11 and LmVP1.1 transcripts and genomic organizations: alternative splicing contributing to the diversity of scorpion venom peptides. Toxicon 2009, 53(1):129-134

34. Valdez-Cruz NA, Batista CV, Zamudio FZ, Bosmans F, Tytgat J, Possani LD: Phaiodotoxin, a novel structural class of insect-toxin isolated from the venom of the Mexican scorpion Anuroctonus phaiodactylus. Eur J Biochem 2004, 271(23-24):4753-4761.

35. Inceoglu B, Lango J, Wu J, Hawkins P, Southern J, Hammock BD: Isolation and characterization of a novel type of neurotoxic peptide from the venom of the South African scorpion Parabuthus transvaalicus (Buthidae). Eur J Biochem 2001, 268(20):5407-5413.

36. Rodriguez de la Vega RC, Possani LD: Overview of scorpion toxins specific for $\mathrm{Na}+$ channels and related peptides: biodiversity, structure-function relationships and evolution. Toxicon 2005, 46(8):831-844.

37. Mouhat S, Andreotti N, Jouirou B, Sabatier JM: Animal toxins acting on voltage-gated potassium channels. Curr Pharm Des 2008, 14(24):2503-2518.

38. Tan PT, Srinivasan KN, Seah SH, Koh JL, Tan TW, Ranganathan S, Brusic V: Accurate prediction of scorpion toxin functional properties from primary structures. J Mol Graph Model 2005, 24(1):17-24.

39. Diego-Garcia E, Abdel-Mottaleb Y, Schwartz EF, de la Vega RC, Tytgat J, Possani LD: Cytolytic and K+ channel blocking activities of beta-KTx and scorpine-like peptides purified from scorpion venoms. Cell Mol Life Sci 2008, 65(1):187-200.

40. Norton RS, McDonough SI: Peptides targeting voltage-gated calcium channels. Curr Pharm Des 2008, 14(24):2480-2491.

41. Bulet $P$, Stocklin $R$, Menin L: Anti-microbial peptides: from invertebrates to vertebrates. Immunol Rev 2004, 198:169-184.

42. Jenssen $H$, Hamill $P$, Hancock RE: Peptide antimicrobial agents. Clin Microbiol Rev 2006, 19(3):491-511.

43. Ehret-Sabatier L, Loew D, Goyffon M, Fehlbaum P, Hoffmann JA, van Dorsselaer A, Bulet P: Characterization of novel cysteine-rich antimicrobial peptides from scorpion blood. J Biol Chem 1996, 271(47):29537-29544.

44. Gennaro R, Zanetti M, Benincasa M, Podda E, Miani M: Pro-rich antimicrobial peptides from animals: structure, biological functions and mechanism of action. Curr Pharm Des 2002, 8(9):763-778.

45. Hancock RE, Brown KL, Mookherjee N: Host defence peptides from invertebrates-emerging antimicrobial strategies. Immunobiology 2006 211(4):315-322.

46. Zeng XC, Wang SX, Zhu Y, Zhu SY, Li WX: Identification and functional characterization of novel scorpion venom peptides with no disulfide bridge from Buthus martensii Karsch. Peptides 2004, 25(2):143-150.

47. Orivel J, Redeker V, Le Caer JP, Krier F, Revol-Junelles AM, Longeon A, Chaffotte A, Dejean A, Rossier J: Ponericins, new antibacterial and insecticidal peptides from the venom of the ant Pachycondyla goeldii. J Biol Chem 2001, 276(21):17823-17829.

48. Zeng XC, Li WX, Peng F, Zhu ZH: Cloning and characterization of a novel cDNA sequence encoding the precursor of a novel venom peptide (BmKbpp) related to a bradykinin-potentiating peptide from Chinese scorpion Buthus martensii Karsch. IUBMB Life 2000, 49(3):207-210.

49. Moerman L, Bosteels S, Noppe W, Willems J, Clynen E, Schoofs L, Thevissen K, Tytgat J, Van Eldere J, Van Der Walt J, et al: Antibacterial and antifungal properties of alpha-helical, cationic peptides in the venom of scorpions from southern Africa. Eur J Biochem 2002, 269(19):4799-4810.
50. Diego-Garcia E, Batista CV, Garcia-Gomez Bl, Lucas S, Candido DM, GomezLagunas F, Possani LD: The Brazilian scorpion Tityus costatus Karsch: genes, peptides and function. Toxicon 2005, 45(3):273-283.

51. Carballar-Lejarazu R, Rodriguez MH, de la Cruz Hernandez-Hernandez F, Ramos-Castaneda J, Possani LD, Zurita-Ortega M, Reynaud-Garza E, Hernandez-Rivas R, Loukeris T, Lycett G, et al: Recombinant scorpine: a multifunctional antimicrobial peptide with activity against different pathogens. Cell Mol Life Sci 2008, 65(19):3081-3092.

52. Soudani N, Gharbi-Chihi J, Srairi-Abid N, Yazidi CM, Planells R, Margotat A Torresani J, El Ayeb M: Isolation and molecular characterization of LVP1 lipolysis activating peptide from scorpion Buthus occitanus tunetanus. Biochim Biophys Acta 2005, 1747(1):47-56.

53. Matsushita N, Miyashita M, Sakai A, Nakagawa Y, Miyagawa H: Purification and characterization of a novel short-chain insecticidal toxin with two disulfide bridges from the venom of the scorpion Liocheles australasiae. Toxicon 2007, 50(6):861-867.

54. Pimenta AM, Stocklin R, Favreau P, Bougis PE, Martin-Eauclaire MF: Moving pieces in a proteomic puzzle: mass fingerprinting of toxic fractions from the venom of Tityus serrulatus (Scorpiones, Buthidae). Rapid Commun Mass Spectrom 2001, 15(17):1562-1572.

55. Abdel-Rahman MA, Omran MA, Abdel-Nabi IM, Ueda H, McVean A: Intraspecific variation in the Egyptian scorpion Scorpio maurus palmatus venom collected from different biotopes. Toxicon 2009, 53(3):349-359.

56. Corzo G, Escoubas P, Villegas E, Barnham K, He W, Norton RS, Nakajima T: Characterization of unique amphipathic antimicrobial peptides from venom of the scorpion Pandinus imperator. Biochem J 2001, 359(Pt 1):35-45.

57. Dai L, Yasuda A, Naoki H, Corzo G, Andriantsiferana M, Nakajima T: IsCT, a novel cytotoxic linear peptide from scorpion Opisthacanthus madagascariensis. Biochem Biophys Res Commun 2001, 286(4):820-825.

58. Chalekson CP, Neumeister MW, Jaynes J: Treatment of infected wounds with the antimicrobial peptide D2A21. J Trauma 2003, 54(4):770-774.

59. Zhao $H$, Wang L, Yuan J: Origin and time of qiongzhou strait. Marine Geology \& Quaternary Geology 2007, 27(2):33-40.

60. Gopalakrishnakone P, Cheah J, Gwee MC: Black scorpion (Heterometrus longimanus) as a laboratory animal: maintenance of a colony of scorpion for milking of venom for research, using a restraining device. Lab Anim 1995, 29(4):456-458.

61. Ewing B, Hillier L, Wendl MC, Green P: Base-calling of automated sequencer traces using phred. I. Accuracy assessment. Genome Res 1998, 8(3):175-185

62. Bendtsen JD, Nielsen $H$, von Heijne $G$, Brunak S: Improved prediction of signal peptides: SignalP 3.0. J Mol Biol 2004, 340(4):783-795

63. Thompson JD, Gibson TJ, Plewniak F, Jeanmougin F, Higgins DG: The CLUSTAL_X windows interface: flexible strategies for multiple sequence alignment aided by quality analysis tools. Nucleic Acids Res 1997, 25(24):4876-4882.

64. Waterhouse AM, Procter JB, Martin DM, Clamp M, Barton GJ: Jalview Version 2-a multiple sequence alignment editor and analysis workbench. Bioinformatics 2009, 25(9):1189-1191.

doi:10.1186/1471-2164-11-452

Cite this article as: Ruiming et al: Comparative venom gland transcriptome analysis of the scorpion Lychas mucronatus reveals intraspecific toxic gene diversity and new venomous components. $B M C$ Genomics 2010 11:452. 